\title{
First demonstration of single trench fiber for delocalization of higher order modes
}

\author{
Deepak Jain ${ }^{1}$, Catherine Baskiotis ${ }^{1}$, Jaesun Kim ${ }^{2}$, and Jayanta K. Sahu \\ 1. Optoelectronic Research Center, University of Southampton, Southampton SO17 1BJ, UK \\ 2. SPI Lasers, Southampton, UK \\ dj3g11@orc.soton.ac.uk
}

\begin{abstract}
We demonstrate an ytterbium-doped single-trench fiber ensuring a high losses ratio $(\sim 1000)$ and low power fraction ( $\sim .7)$ between the higher-order-modes and fundamental-mode with excellent bend robustness and $85 \%$ laser efficiency at a wavelength of $1040 \mathrm{~nm}$.

OCIS codes :(060.2280) Fiber design and manufacturing; (140.3510) Fiber lasers.
\end{abstract}

\section{Introduction}

Novel large mode area (LMA) fiber designs have led to significant progress in the development of fiber lasers in the past years. For high power applications, a LMA fiber design should offer characteristics like high suppression of the higher order modes (HOMs), low bending losses of the fundamental mode (FM), and easy fabrication. Several fiber designs such as low NA step index fibers (SIFs) [1], Bragg fibers [2], 2-D all solid photonic bandgap fibers (2DASPBGFs) [2-3], and photonic crystal fibers (PCFs) based designs like leakage channel fibers (LCFs) [4] have been proposed to scale the effective area $\left(\mathrm{A}_{\text {eff }}\right)$ of the FM. However, most of these designs require refractive index of the core to be equal to the cladding which poses serious challenge in fabricating rare earth ions doped core having refractive index equal to the cladding. Moreover, PCFs and 2D-ASPBGFs require stack and draw technique. Recently, we proposed multi trench fibers (MTFs), which are easy to fabricate and can provide very high suppression of the HOMs [5]. In this paper, we demonstrate for the first time, a ytterbium ( $\mathrm{Yb}$ )-doped single trench fiber which is having refractive index of the core higher than the cladding and offers very high suppression of the HOMs with easy fabrication requirements. The fiber exhibited a laser efficiency of $\sim 85 \%$ at a wavelength of 1040 $\mathrm{nm}$ whilst retaining good beam quality.

\section{Numerical Simulations}

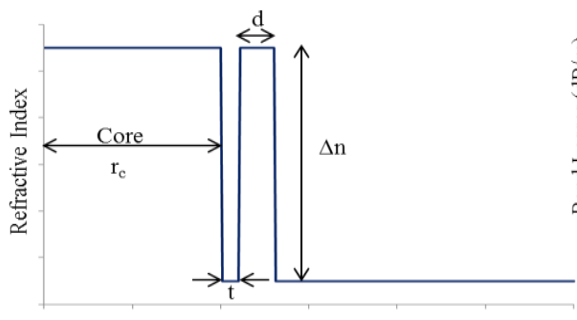

(a)

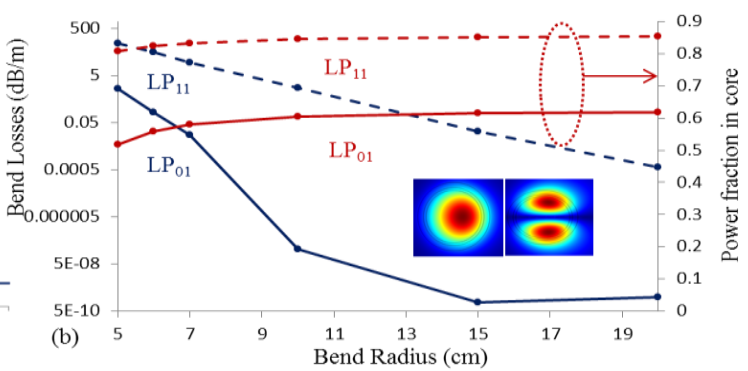

Fig. 1(a) Schematic cross-section of the proposed single-trench fiber. (b) Computed bend losses and power fraction of the $\mathrm{FM}$ and $\mathrm{LP}_{11}$ mode for different bend radius. Inset shows the images of the FM and $\mathrm{LP}_{11}$ mode at $7 \mathrm{~cm}$ bend radius.

Fig. 1(a) presents the schematic of single trench fiber. Fig. 1(b) shows the numerically computed bend losses of the $\mathrm{FM}$ and $\mathrm{LP}_{11}$ mode (here $\mathrm{LP}_{11}$ has lowest losses and highest power fraction among all other HOMs). The fiber parameters considered in our simulations are $r_{c}=10 \mu \mathrm{m}, \mathrm{t}=1 \mu \mathrm{m}, \Delta \mathrm{n}=0.001$, and $\mathrm{d}=2 \mu \mathrm{m}$. The bending losses of the $\mathrm{LP}_{11}$ mode are larger than $18 \mathrm{~dB} / \mathrm{m}$ and for the FM mode it is lower than $0.012 \mathrm{~dB} / \mathrm{m}$ at $7 \mathrm{~cm}$ bend radius, ensuring a losses ratio of the HOMs to FM larger than $10^{3}$ and the $A_{\text {eff }}$ is $\sim 340 \mu \mathrm{m}^{2}$. Moreover, power fraction ratio of the HOMs to the FM in core maintains a good ratio of $\sim 0.7$ irrespective of bend radius. It is important to note that, in a double clad fiber laser, where all the effective losses are zero, low power fraction of the HOMs in core compared to the FM is an important factor to ensure single mode operation. Due to this low power fraction of the HOMs compared to the FM in core, a single mode output can be ensured irrespective of the bend radius thanks to the delocalization of the $\mathrm{LP}_{11}$ mode. Moreover, numerical simulations show that two and three trench fibers can achieve much larger $A_{\text {eff }}\left(600-700 \mu \mathrm{m}^{2}\right)$ with similar level of suppression, although a compromise with bend radius has to be made [6]. The delocalization of the $\mathrm{LP}_{11}$ mode is due to presence of the resonant ring surrounding the core. The best suppression of the HOMs that can be achieved from a SIF with the same core radius $\left(r_{c}=10 \mu \mathrm{m}\right)$ and refractive index contrast $(\Delta \mathrm{n}=0.001)$ is only a losses ratio of $\sim 280$ and power fraction ratio larger than 0.82 with $\mathrm{A}_{\text {eff }}$ of $\sim 340 \mathrm{um}^{2}[1$, 6]. Moreover, in case of single trench fiber the level of HOMs suppression and the operational range of bending radius is better than other fiber designs like PCFs, W-type fibers, Parabolic fiber etc. [5-7]. 


\section{Experimental Realization of Yb-doped Single Trench Fiber}

Fig. 2(a) shows the measured refractive index profile (RIP) of $\mathrm{Yb}^{3+}$ and $\mathrm{Al}^{3+}$ doped preform fabricated using conventional MCVD and solution doping technique. Preform was milled to D-shaped and drawn to an outer diameter of $240 \mu \mathrm{m}$ (core diameter $20 \mu \mathrm{m}$ ) with low index polymer coating. The fiber shows $2.5 \mathrm{~dB} / \mathrm{m}$ absorption at $975 \mathrm{~nm}$. Fig. 2 (b) shows the measured Multi-Path Interference (MPI) versus group delay using $\mathrm{S}^{2}$ measurement. $\mathrm{LP}_{11}$ and $\mathrm{LP}_{21}$ modes suffer high losses and their power level is $32 \mathrm{~dB}$ lower than the FM. A significant amount of power for the HOMs is observed outside the core which ensures delocalization of the HOMs. A $6.17 \mathrm{~m}$ of fiber was used in a 4\%-4\% linear laser cavity to measure the laser efficiency. Fig. 3 (a) shows the output spectrum of fiber laser at the bend radius of $5 \mathrm{~cm}$ and $7.5 \mathrm{~cm}$. Inset shows the captured output beam image for $5 \mathrm{~cm}$ bend case. Both lasers show lasing at a wavelength 1040nm wavelength. Fig. 3(b) shows the output power of the fiber laser for different bend radius. The slope efficiency measured is approximately $85 \%$ with respect to the launched pump power with $\mathrm{M}^{2} \sim 1.3$.

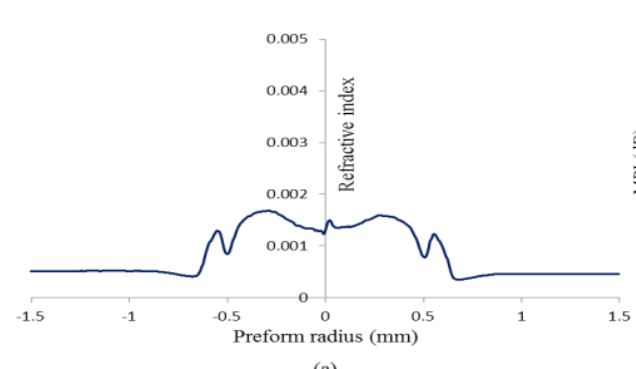

(a)

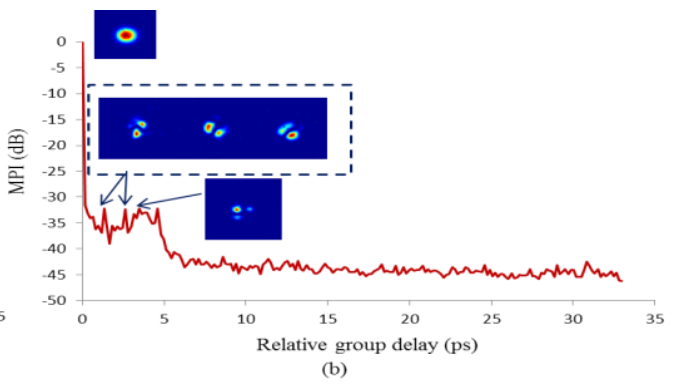

(b)

Fig. 2(a) Measured RIP of fabricated preform (b) MPI (dB) versus relative group delay (ps) for fabricated fiber. Inset images show the beam profiles of obtained modes.

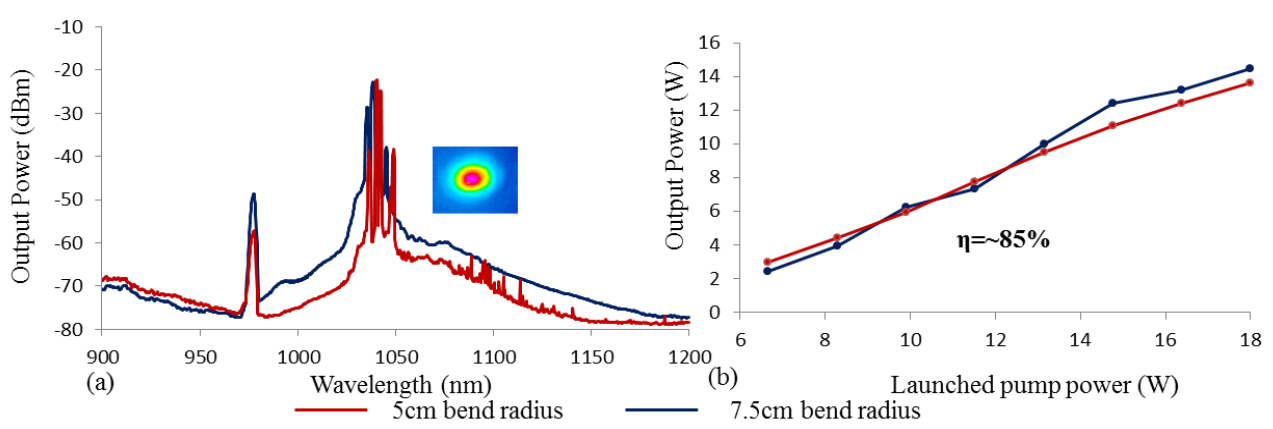

Fig. 3 (a) Measured spectrum of the output beam (b) slope efficiency for two different bend radius of fiber. Inset shows the output beam for fiber laser bent at $5 \mathrm{~cm}$ radius (shown in red colour).

\section{Conclusion}

We have demonstrated, for the first time, an efficient Yb-doped single trench fiber in the MTF family. The fiber ensures a single mode operation by offering large losses and poor power fraction of the HOMS in the core thanks to the delocalization of HOMs. The laser yields output at $1040 \mathrm{~nm}$ with $\sim 85 \%$ slope efficiency from a $20 \mu \mathrm{m}$ core fiber. Our proposed fiber design is suitable for mass production using conventional low-loss fiber fabrication techniques.

\section{References}

[1] M.-J. Li et. al., "Limit of effective area for single-mode operation in step-index large mode area laser fibers," J. Lightw. Technol., vol. 27, pp. 3010-3016, August 2009.

[2] E. M. Dianov et. al., "Solid-Core photonic bandgap fibers for high-power fiber lasers," IEEE J. Sel. Top. Quantum Electron., vol. 15, 2009.

[3] F. Kong et. al., "Mode area scaling with all-solid photonic bandgap fiber," Opt. Exp., vol. 20, no. 24, pp. 26363-26372, Nov. 2012.

[4] L. Dong et. al, "Ytterbium-doped all glass leakage channel fibers with highly fluorine-doped silica pump cladding", Opt. Exp., vol. 17, pp. 8962-8969, May 2009.

[5] D. Jain et. al., "Bending performance of large mode area muli-trench fibers," Opt. Exp. vol. 21, pp. 26663-26670, Oct. 2013.

[6] D. Jain et. al., "Large mode area multi-trench fiber with delocalization of higher order modes [Invited]," to be published in Special issue on high power fiber lasers, IEEE J. Sel. Top. Quant. Elect., Sep. 2014.

[7] J. M. Fini, "Bend-resistant design of conventional and microstructure fibers with very large mode area," Opt. Exp., vol. 14, pp. 69-81, Jan. 2006.

\section{Acknowledgement}

The work is supported by the EPSRC Centre for the Innovative manufacturing in Photonics EP/HO2607X/1. 